\title{
Direct detection of galaxy stellar halos: NGC 3957 as a test case ${ }^{\star}$
}

\author{
P. Jablonka ${ }^{1,2,3}$, M. Tafelmeyer ${ }^{1}$, F. Courbin ${ }^{1}$, and A. M. N. Ferguson ${ }^{4}$ \\ ${ }^{1}$ Laboratoire d'Astrophysique, Ecole Polytechnique Fédérale de Lausanne (EPFL), Observatoire, 1290 Sauverny, Switzerland \\ e-mail: pascale.jablonka@unige.ch \\ 2 Université de Genève, Observatoire, 1290 Sauverny, Switzerland \\ 3 Observatoire de Paris, CNRS-UMR8111, Place Jules Janssen, 92190 Meudon, France \\ ${ }^{4}$ Institute for Astronomy, University of Edinburgh, Blackford Hill, Edinburgh, EH9 3HJ, UK
}

Received 18 September 2009 / Accepted 18 January 2010

\section{ABSTRACT}

\begin{abstract}
We present a direct detection of the stellar halo of the edge-on S0 galaxy NGC 3957, using ultra-deep VLT/VIMOS $V$ and $R$ images. This is achieved with a sky subtraction strategy based on infrared techniques. These observations allow us to reach unprecedented high signal-to-noise ratios of up to $15 \mathrm{kpc}$ away from the galaxy center, rendering photon-noise negligible. The $1 \sigma$ detection limits are $R=30.6 \mathrm{mag} / \operatorname{arcsec}^{2}$ and $V=31.4 \mathrm{mag} / \mathrm{arcsec}^{2}$. We conduct a thorough analysis of the possible sources of systematic errors that could affect the data: flat-fielding, differences in CCD responses, scaling of the sky background, the extended halo itself, and PSF wings. We conclude that the $V-R$ colour of the NGC 3957 halo, calculated between 5 and $8 \mathrm{kpc}$ above the disc plane where the systematic errors are modest, is consistent with an old and preferentially metal-poor normal stellar population, like that revealed in nearby galaxy halos from studies of their resolved stellar content. We do not find support for the extremely red colours found in earlier studies of diffuse halo emission, which we suggest might have been due to residual systematic errors.
\end{abstract}

Key words. galaxies: halos - galaxies: stellar content

\section{Introduction}

Galaxy stellar halos contain fundamental clues about the galaxy assembly. $\Lambda \mathrm{CDM}$ models predict stellar halos to be ubiquitous and dominated by old metal-poor populations, characterized by significant substructures in the form of tidal debris from accreted satellites (Bullock \& Johnston 2005; Abadi et al. 2006; Font et al. 2006). Conversely, in classical dissipative collapse models, halos are expected to exhibit smooth age and metallicity gradients (e.g., Eggen et al. 1962). Due to the extreme faintness of these envelopes, the value of galactic stellar halos as key tests of galaxy formation theories has however not yet been fully realized.

Since the middle of the nineties, our view of galaxy haloes has changed dramatically with the discovery of substructures around the Milky Way (e.g., Ibata et al. 1994; Majewski et al. 1999; Newberg et al. 2002; Gilmore et al. 2002; Rocha-Pinto et al. 2003; Vivas \& Zinn 2006; Grillmair 2006; Belokurov et al. 2007; Jurić et al. 2008; Bell et al. 2008). Similar signatures of tidal destruction of satellites by their massive hosts were found in the halo in M 31 (e.g., Ibata et al. 2001; Ferguson et al. 2002; Ibata et al. 2007; Richardson et al. 2008; McConnachie et al. 2009; Tanaka et al. 2010). Stellar streams were also detected around NGC 5907 and NGC 4013 (Martínez-Delgado et al. 2008, 2009). It is nevertheless not yet totally clear how ubiquitous halos are and how often mergers intervene in their building-up.

Resolving individual stars is mainly limited to a small sample of galaxies within or close to the Local Group, with a few

^ Based on observations obtained at the ESO Very Large Telescope (VLT) in the Program 077.B0145(A). exceptions. Mouhcine et al. (2005a,b) resolved the stars near the red giant branch tip in the outskirts of eight nearby galaxies with the HST/WFPC2, although it remains unclear for some of their fields whether they sampled the galaxy outer discs or halos. Similarly, Mouhcine et al. (2007); Ibata et al. (2009); Rejkuba et al. (2009) analysed the extra-planar stellar populations in NGC 891 using three HST/ACS pointings. Recently Barker et al. (2009) conducted the first wide-field ground-based survey of the red giant branch population in the outskirts of M 81 using Suprime-Cam on the 8-m Subaru telescope. They detected a faint, extended structural component beyond the galaxy's bright optical disc sharing some similarities with the Milky Way's halo, but also exhibiting some important differences.

Searches for extended low surface brightness diffuse halo emission were carried out around several more distant external galaxies. The first detection was announced by Sackett et al. (1994) around the edge-on Sc galaxy NGC 5907 and later confirmed by Lequeux et al. (1996) and Lequeux et al. (1998). These two subsequent works additionally reported an unexpected reddening of the halo colours, increasing with the distance to the galaxy center. These results were contested by Zheng et al. (1999), who noticed that artifacts in the star-masking procedure could be responsible for the apparent reddening. Zibetti et al. (2004) used a stacking technique to combine the images of 1047 edge-on galaxies selected from the Sloan Digital Sky Survey. They detected the presence of a mean luminous halo, whose shape was clearly rounder than the disc. Their data suggested a correlation between the halo and galaxy luminosities. Surprisingly, their halo colours were redder than the reddest known elliptical galaxies. Zibetti \& Ferguson (2004) reached similar photometric depths in a study of a single edge-on galaxy 
in the Hubble Ultra Deep Field, where they also found anomalously red colours, not reproduced by any conventional stellar populations model and getting redder with larger radii. de Jong (2008) however discussed how scattered galaxy light from extended point spread function tails could have been underestimated in these studies and thus lead to spurious detections.

The controversy over the existence and nature of stellar halos around external galaxies reflects that these types of observations are extremely challenging from a technical standpoint. Errors in flat-fielding and sky subtraction can easily mask or contort real signal. In order to overcome these limitations and to understand galactic halo properties, we designed a new strategy for galactic halo surface brightness profiles. It is based on two principles: i) we target a galaxy, which has an angular size that is much larger than the PSF extent and ii) we carry out very accurate sky subtraction, with a chopping technique similar to that used in near-IR imaging.

This paper is organized as follows: Sect. 2 presents our observational technique. Section 3 describes the data reduction procedure leading to ultra-deep sky-subtracted $V$ and $R$ images of NGC 3957. Section 4 presents the radial surface brightness profiles derived for the halo of NGC 3957. Section 5 discusses the most important possible sources of errors, focusing on the systematics. Section 6 interprets the $V-R$ colour profile. Our conclusions are summarized in Sect. 7.

\section{Observational strategy}

We selected the early-type (S0) galaxy NGC 3957 (RA (J2000): 1154 01.5; Dec (J2000): -1934 08; $v_{\text {hel }}=$ $\left.1637 \mathrm{~km} \mathrm{~s}^{-1}\right)$. NGC 3957 is seen nearly edge-on, its exact inclination angle $(88 \pm 1$ degrees) was measured by Pohlen et al. (2004). Its apparent size $\left(3.1^{\prime} \times 0.7^{\prime}\right)$ is large enough to allow us to integrate flux over large regions when determining the surface brightness profiles. At the same time, it is small enough to fall entirely in a single VIMOS CCD chip. Taking $H_{0}=73 \mathrm{~km} \mathrm{~s}^{-1} \mathrm{Mpc}^{-1}$, at the distance of NGC 3957 $(22.42 \mathrm{Mpc}), 1 \mathrm{arcmin}=6.52 \mathrm{kpc}$. Another important point is that the surroundings of NGC 3957, which were used to determine the background level and shape, are devoid of any bright objects.

The choice of VIMOS was motivated by the opportunity to have a wide field of view distributed over four different CCDs, of $2048 \times 2440$ pixels each. With a resolution of $0.205^{\prime \prime}$ per pixel, each CCD covers $7^{\prime} \times 8^{\prime}$. They are separated by a gap of $2^{\prime}$ (see Fig. 2). Unfortunately, the choice of a guide star satisfying VIMOS specifications had the consequence that the guide probe fell on two of the four CCDs. In all data, it covers more than $50 \%$ of either CCD3 or CCD4 (see CCD4 in Fig. 2), leaving those two chips unusable for our analysis.

We gathered $R$ and $V$-band images, with total exposures of $22500 \mathrm{~s}$ and $25080 \mathrm{~s}$ (i.e., $\sim 6$ and $7 \mathrm{~h}$ ), respectively. Exposures were split into sequences of $190 \mathrm{~s}(R$-band) and $450 \mathrm{~s}$ ( $V$-band). The journal of the observations is presented in Table 1 .

The design of VIMOS allowed us to place NGC 3957 in one CCD while integrating the sky on the other one. This strategy offered two major advantages: (i) On one hand, the background level was estimated in a region located far away from the galaxy, thus avoiding possible contamination by faint, extended galaxy components. (ii) On the other hand, a 2D image of the sky was obtained under the exact same observing conditions as those of the galaxy.

Exposures were dithered following the patterns shown in Fig. 1. This allowed us not only to build master skies from empty
Table 1. Journal of the observations.

\begin{tabular}{ccc}
\hline \hline $\begin{array}{c}\text { Date } \\
(\mathrm{jjjj} / \mathrm{mm} / \mathrm{dd})\end{array}$ & Exposure & Filter \\
\hline $2006 / 04 / 24$ & $24 \times 190 \mathrm{~s}$ & $R$ \\
$2006 / 04 / 25$ & $24 \times 190 \mathrm{~s}$ & “" \\
$2006 / 04 / 27$ & $12 \times 190 \mathrm{~s}$ & “ \\
$2006 / 04 / 28$ & $24 \times 190 \mathrm{~s}$ & “ \\
$2006 / 04 / 29$ & $24 \times 190 \mathrm{~s}$ & “ \\
$2006 / 05 / 25$ & $24 \times 190 \mathrm{~s}$ & “ \\
\hline $2006 / 05 / 27$ & $12 \times 450 \mathrm{~s}$ & $V$ \\
$2006 / 05 / 28$ & $12 \times 450 \mathrm{~s}$ & “ \\
$2006 / 05 / 29$ & $12 \times 450 \mathrm{~s}$ & “ \\
$2006 / 05 / 31$ & $14 \times 450 \mathrm{~s}$ & “ \\
\hline
\end{tabular}

Notes. Half of the exposures of each night were taken with the galaxy located in CCD1 and the others with the galaxy in CCD2 (see Fig. 2).

field frames, nicely removing any bright object, but also to smear out any inhomogeneities in the background, increasing the flatness of the combined master skies.

As already mentioned, we used two different pointings. We will use the following terminology throughout: Pointing 1 corresponds to a set of exposures placing the galaxy in CCD1 and dithered following the patterns of Fig. 1. Pointing 2 has the same dithering pattern as Pointing 1, but the galaxy is now placed in CCD2. The exposures on the empty field in CCD1 (when the galaxy is in CCD2) and CCD2 (when the galaxy is in CCD1) are used to determine the background level and shape.

\section{Data reduction and sky subtraction}

We only describe at length the steps differing from the classical methodology for the reduction of images. These steps are critical to reach a surface brightness level down to $\sim 30 \mathrm{mag} / \mathrm{arcsec}^{2}$.

Bias and flat-field corrections were done in a classical manner. Five featureless biases were taken each night. Their mean ADU/pixel was subtracted from all frames of the same night. When possible, a series of five twilight flat fields were taken. For each of these nights, we composed a master flat, corresponding to the average of the five flats, with a 2- $\sigma$ clipping boundary, $\sigma$ being calculated with the gain and readout noise of the CCDs. Since flat-fields were not taken each night, the same master flat was used for several subsequent nights. We will discuss the possible uncertainties arising from the flat-fielding in Sect. 5.1.

The most crucial step of the data reduction is the sky subtraction. One master sky per night and per CCD was created as the average (plus 2- $\sigma$ clipping boundary) of all dithered frames which did not contain the galaxy. For the $R$-band, 12 sky images per night and CCD were available. Sigma clipping of the dithered images proved sufficient to remove any bright star in the field. For the $V$-band, only six frames were available. Consequently, we had to mask the bright stars before dithering to remove them completely from the master sky frames. Figure 3 provides an example of a master sky for the two photometric bands. The cuts are chosen to be $\pm 2 \%$ of the mean background level, which is three times the standard deviation. The large scale structures represent $\lesssim 1 \%$ of the mean background level. They are of the order of the uncertainties in flat-fielding, which are discussed in Sect. 5.1.

For the sake of clarity, we will now use the following nomenclature (see Fig. 4):

- MS1: master sky of CCD1, taken from the empty field frames of Pointing 2; 


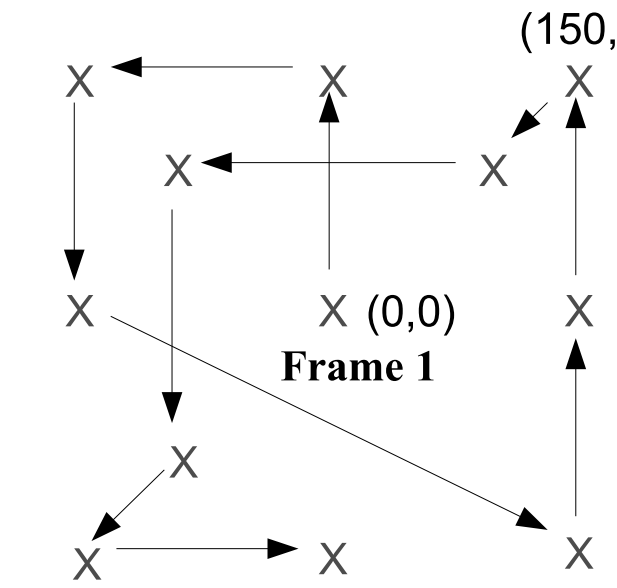

$(-150,-150)$ Frame 12

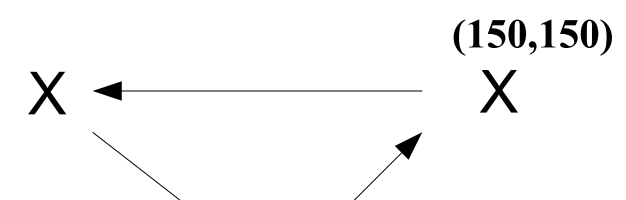

$(0,0) X$

Frame 1

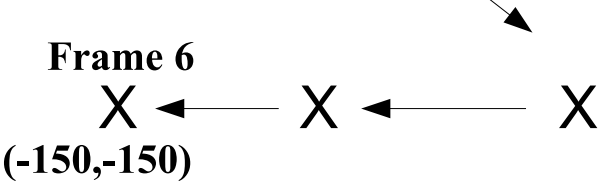

Fig. 1. Left: dithering pattern for a set of 12 consecutive $R$-band frames. The position $(0,0)$ indicates the initial position of NGC 3957 in one CCD. Right: dithering pattern for a set of six consecutive $V$-band exposures. Units are given in pixels. One pixel is $0.205^{\prime \prime}$.
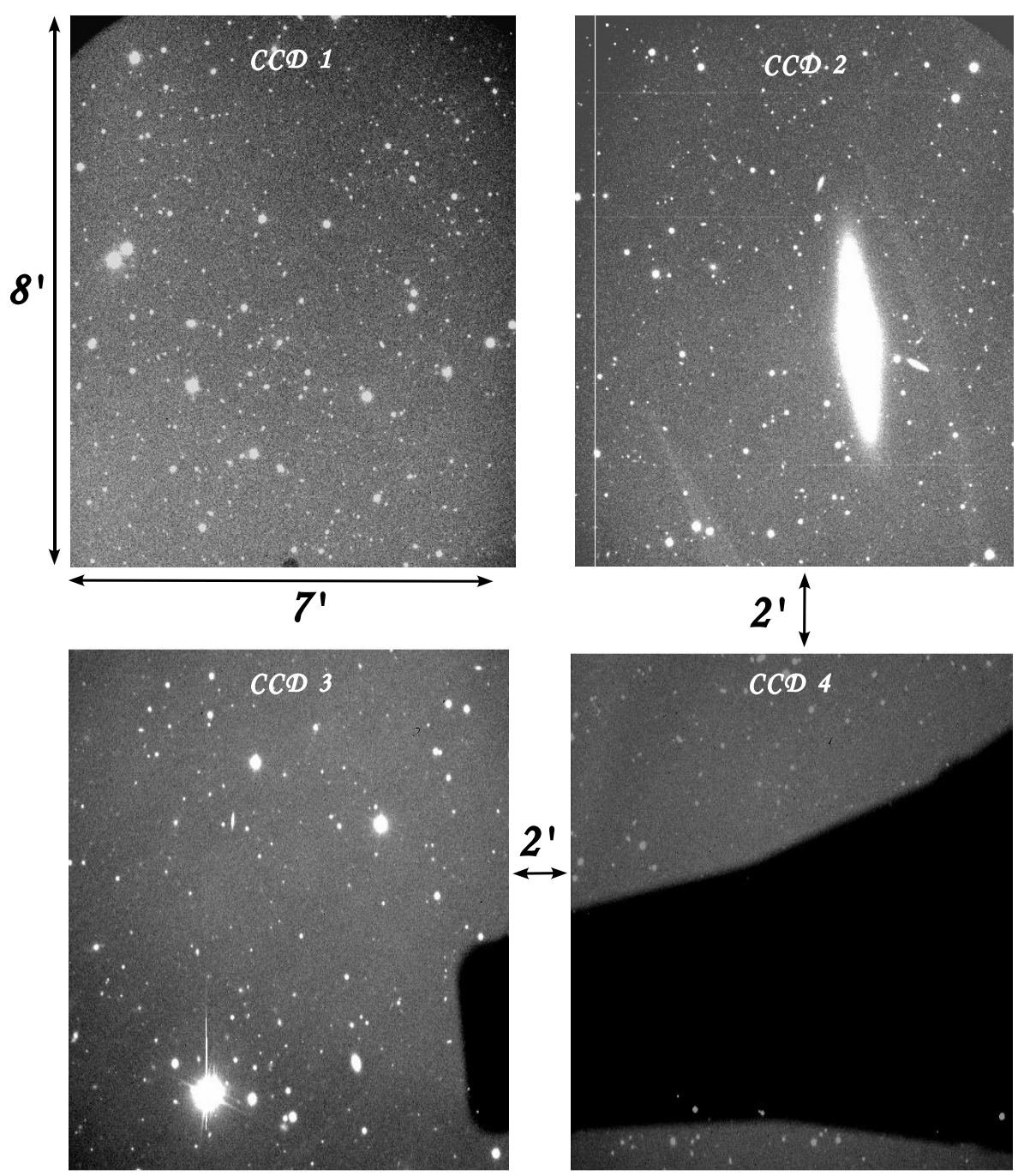

Fig. 2. Geometry of the VIMOS field of view, composed of four CCDs, prior to sky subtraction. The galaxy was alternately put in CCD1 and in CCD2. The black feature in CCD3 and $\mathrm{CCD} 4$ is due to the guide star camera.

- SKY1 $1_{\mathrm{i}}$ : individual CCD1 image of Pointing 2, which does not contain the galaxy and from which MS1 was created;

- GAL1 $1_{\mathrm{i}}$ : individual CCD1 image of Pointing 1, which contains the galaxy;
- MS2: master sky of CCD2 with Pointing 1. Analogous to MS1;

- SKY2 $2_{\mathrm{i}}$ : individual CCD2 image of the empty field with Pointing 1. Analogous to SKY $1_{i}$; 

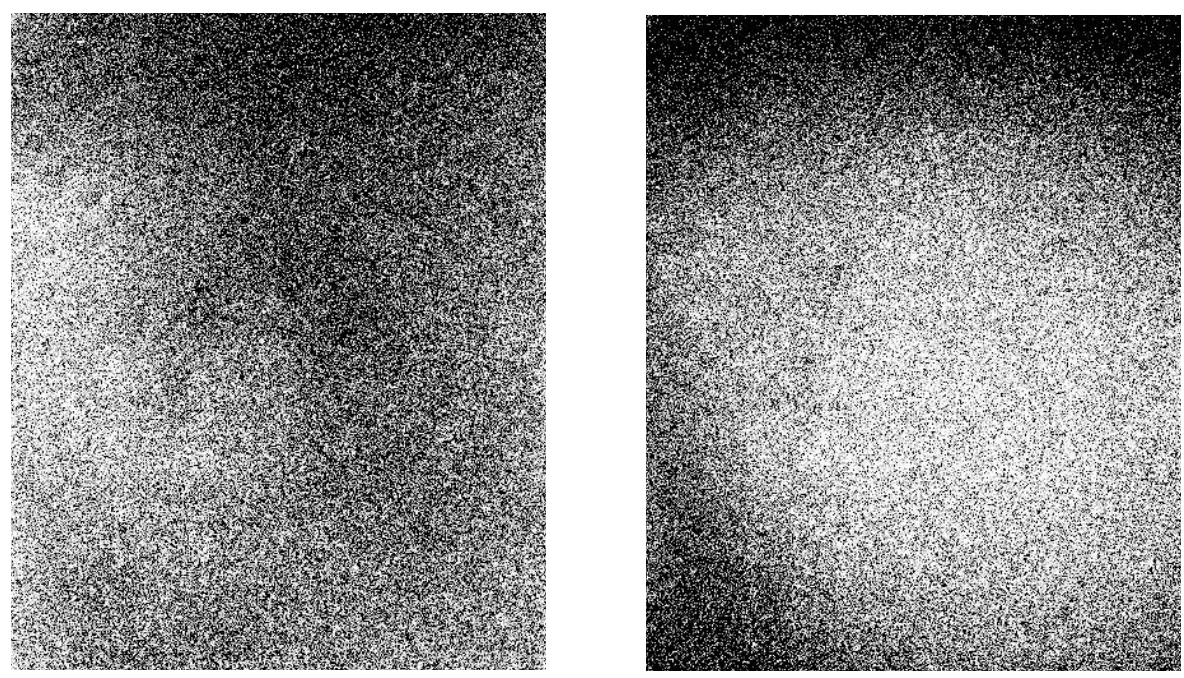

Fig. 3. Example of the master sky in $R$ (left panel) and $V$ (right panel). The cuts are $\pm 3 \sigma$, the large scale variations are $\lesssim 1 \%$.

- GAL2 ${ }_{\mathrm{i}}$ : CCD2 individual galaxy image of CCD2 with Pointing 2. Analogous to $\mathrm{GAL}_{1}$.

The index i goes from 1 to 12 for the $R$-band or from 1 to 6 for the $V$-band.

We could not directly use the sky frames obtained at the same time as the galaxy. Indeed, galaxy and background would then have been observed with different CCDs. Small differences in the flat-fielding between CCD1 and CCD2 could introduce artificial intensity gradients in the final images. We needed to subtract skies of Pointing 2 from the galaxy frames of Pointing 1 (and vice versa) obtained during the same night. In practice, for each night we subtracted MS1 from GAL1 $1_{i}$ and MS2 from GAL2 $2_{i}$, i.e. the master skies were observed at a slightly different time than the galaxy, but with the same CCD. Although full dithering patterns for both pointings were completed in about one hour, the sky level was not necessarily stable in this time interval. Actually, we measured a variation in the mean background level of $10-15 \%$ from the first to the last exposure for a given pointing sequence. Two corrections were therefore necessary:

(1) For each GAL1 $1_{i}\left(G A L 2{ }_{i}\right)$, MS1(MS2) had to be scaled to the level of the sky at the time at which the galaxy was observed. We had direct access to this information via the contemporaneous exposures $S K Y 2_{i}\left(S K Y 1_{i}\right)$. To determine the scaling factor of each $\mathrm{GAL} 1_{i}, \mathrm{MS} 1$ was multiplied by the ratio

$\beta_{\mathrm{i} 1}=\frac{\left\langle\mathrm{SKY} 2_{\mathrm{i}}\right\rangle}{\langle\mathrm{MS} 1\rangle}$,

where $\langle\mathrm{MS} 1\rangle$ is the mean intensity, in ADU/pixel, of a large central region $(\approx 2 \mathrm{M}$ pixels, i.e., $22 \mathrm{kpc}$-radius region centered at $\sim 50 \mathrm{kpc}$ from the galaxy center) of the master sky frame and $\left\langle\mathrm{SKY} 2_{\mathrm{i}}\right\rangle$ is the mean intensity in the same region of the sky field frame, observed simultaneously with GAL $1_{\mathrm{i}}$. Our surface-brightness limit is $\sim 31.4 \mathrm{mag} / \mathrm{arcsec}^{2}$ in $V$ and $\sim 30.6 \mathrm{mag} / \operatorname{arcsec}^{2}$ in $R$. These limiting magnitudes correspond to the integrated sky noise in $1 \operatorname{arcsec}^{2}$, or equivalently, to the fluxes leading to a signal-to-noise ratio of 1 $\left(28.2 \mathrm{mag} / \operatorname{arcsec}^{2}\right.$ in $R$ and $29.0 \operatorname{arcsec}^{2}$ in $V$ at $S / N=3$ ). Irwin et al. (2005) report a $V$-band halo surface brightness in M 31 of 31-32 mag/ $\operatorname{arcsec}^{2}$ between $\sim 40$ and $55 \mathrm{kpc}$ along the minor axis. Since this area of M 31 is contaminated by tidal debris streams, the actual surface brightness of the underlying halo is somewhat lower (e.g. Ibata et al. 2007). Assuming that the halo of M 31 is identical to that of NGC 3957, it corresponds to the level of noise in our sky frames. (2) Since $\left\langle\mathrm{SKY} 2_{\mathrm{i}}\right\rangle$ and $\langle\mathrm{MS} 1\rangle$ are measured on two distinct CCDs, we had to correct $\beta$ for their different responses. It was done by calculating the ratios of the intensities of the bias-subtracted, non-normalised flatfields of CCD1 and CCD2. Namely, each $\beta_{\mathrm{i}}$ had to be multiplied by a sensitivity correction

$\alpha=\frac{\langle\mathrm{FF} 1\rangle}{\langle\mathrm{FF} 2\rangle}$,

for the scaling of the MS1 frames and by $\alpha^{-1}$ for the scaling of MS2.

Errors in $\alpha$ have opposite effects in CCD1 and CCD2 respectively, leading to a systematic additive offset in the results when considering each of the two CCDs separately. This can be used to fine-tune $\alpha$ removing any systematic difference between CCD1 and CCD2.

The complete sky subtraction is finally described by

$\mathrm{FIN}_{1}=\mathrm{GAL}_{\mathrm{i}}-\mathrm{MS} 1 \cdot \alpha \cdot \beta_{\mathrm{i} 1}$

and

$\mathrm{FIN} 2_{\mathrm{i}}=\mathrm{GAL} 2_{\mathrm{i}}-\mathrm{MS} 2 \cdot \frac{\beta_{\mathrm{i} 2}}{\alpha}$

where $F I N 1_{i}$ and FIN2 $2_{i}$ are the final sky subtracted frames of CCDs 1 and 2. A schematic view of the scaling method is shown in Fig. 4.

The 132 (50) $R$-band ( $V$-band) background-subtracted images were finally aligned and combined, and the final images are calibrated in the Johnson-Cousins photometric system. One CCD frame per night was calibrated in $V$ and $R$ with the standard stars observed during the same period. The final $V$ and $R$ combined images were then scaled to the level of their respective reference $V$ and $R$ frames. Hence, the final frames were multiplied by the factor flux(single)/flux(combined), where flux(single) and flux(combined) were measured from several bright stars in the reference and combined frames, respectively. These photometrically calibrated images were then corrected for extinction using $A_{R}=0.124$ and $A_{V}=0.153$ following Schlegel et al. (1998). The extinction varies by $0.006 \mathrm{mag}$ rms within a radius of 6 arcmin around NGC 3957. Considering random errors alone, the $1-\sigma$ final accuracy of our calibration is 0.022 mag, in $R$, and $0.036 \mathrm{mag}$, in $V$. 


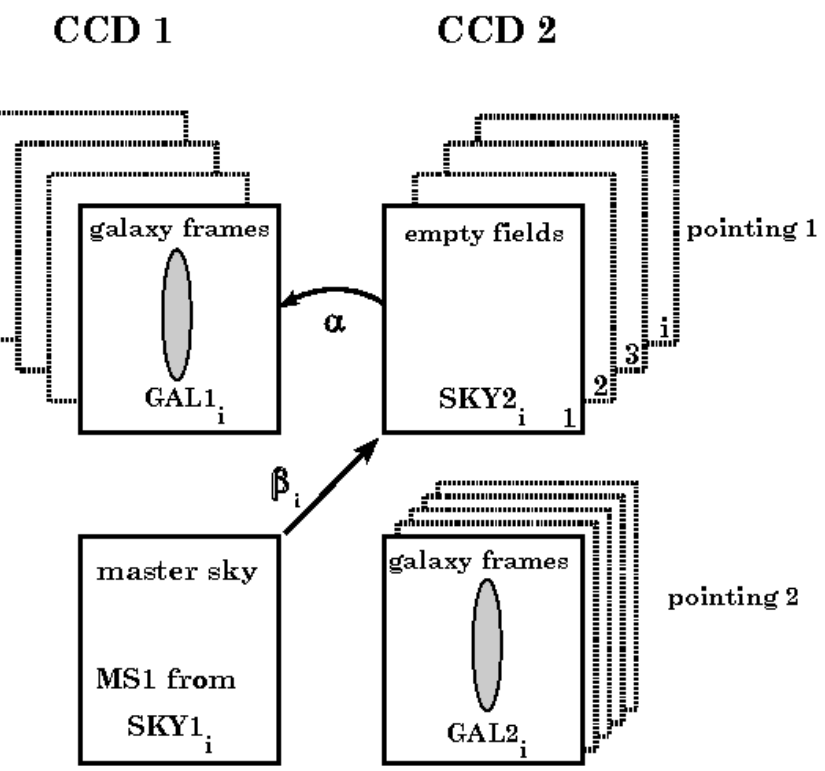

Fig. 4. Explanation of the scaling scheme, prior to the image coaddition. The master sky of CCD1 (MS1) is taken as a reference. The $i$ index refers to the individual exposures within a sequence. The factors $\beta_{\mathrm{i}}$ scale MS1 to match the sky level of each empty fields of CCD2 (SKY $2_{\mathrm{i}}$ ), observed on the same night. The factor $\alpha$ corrects for the difference in sensitivity between the two CCDs. It is determined from the intensity ratios of the flatfields of CCD1 and CCD2.

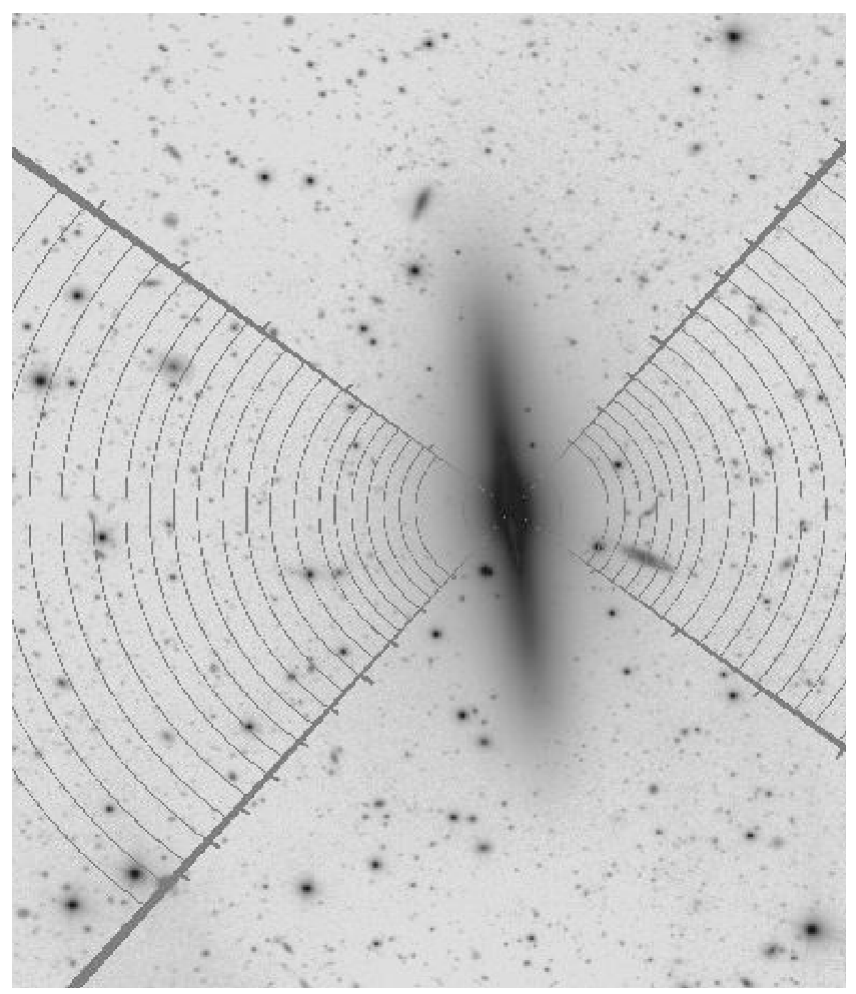

Fig. 5. Cone shaped regions used to integrate the light on the final coadded $V$ and $R$ images. North is to the top, east to the right.

\section{Surface brightness profiles}

In order to trace the galaxy signal down to very faint levels with a sufficiently high signal-to-noise ratio, we integrated flux in $90^{\circ}$ wedges, running along the minor axis of the galaxy (Fig. 5). Prior to this binning, the foreground stars were carefully masked,

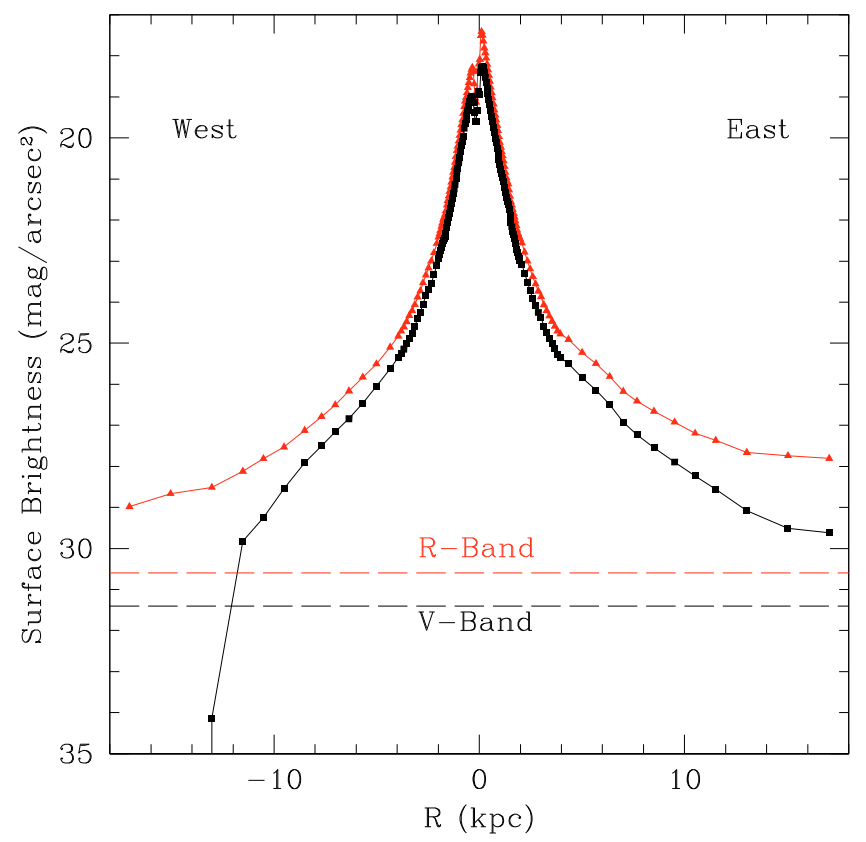

Fig. 6. $V$ - (black) and $R$-band (red) surface brightness profiles along the minor-axis of NGC 3957 . Errors due to photon noise are typically $\leq 0.05 \mathrm{mag}$ within $10 \mathrm{kpc}$ and $\leq 0.1 \mathrm{mag}$ for $r \geq 10 \mathrm{kpc}$, hence too small to be plotted. The systematic errors are not displayed for clarity and can be seen in Fig. 8. The dashed lines indicate the $1 \sigma$ detection limits in the two bands.

as the background galaxies. The radial width of each bin was chosen as a trade-off between the spatial resolution of the galaxy profile and the desired signal-to-noise per bin. The narrowest bins have an annular width of 2 pixels $(0.04 \mathrm{kpc})$ and are located in the central parts of the galaxy, while the widest ones have a radial extent of 100 pixels $(1 \mathrm{kpc})$. The resulting surface brightness profiles in $V$ and $R$-bands, obtained by taking the average of the flux in all unmasked pixel in each radial ring, are shown in Fig. 6, where the dashed lines indicate the detection limits per square arcsecond. The spatial sampling is five times finer than that of Zibetti et al. (2004) in the inner galactic regions, while in the outer parts, the spatial sampling of the two studies is the same. However, unlike Zibetti et al. (2004), who get a signal-tonoise of 1 for the outermost bins at $\sim 12-14 \mathrm{kpc}$, we are not limited by photon noise in these parts $(S / N \sim 20)$. Systematic errors (see Sect. 5) start dominating over photon noise at $r=8-10 \mathrm{kpc}$, where our $S / N$ is still $\approx 40$ in $V$ and $R$.

We estimated the photon noise $\sigma_{\text {tot }}$ per bin by adding in quadrature the photon noise of all $N$ pixels in a cone-shape bin

$\sigma_{\text {tot }}^{2}=\sum_{i}^{N} \sigma_{i}^{2}$,

where the sum goes over all non-masked pixels. The noise per pixel, $i$, on the sky-subtracted and coadded frame is

$\sigma_{i}=\sqrt{\frac{F_{i}}{g}+\sigma_{\text {sky }}^{2}}$,

where $F_{i}$ is the flux in pixel $i, g$ is the electron-to-adu conversion factor, and $\sigma_{\text {sky }}$ is the standard deviation of the pixels in the sky background. This calculation gives typical error bars of $0.05 \mathrm{mag}$ in regions located at about $10 \mathrm{kpc}$ from the centre. The error bars remain smaller than 0.1 mag from $10 \mathrm{kpc}$ to the edges of the frames at $15 \mathrm{kpc}$. These small error bars are too small to be 
seen in Fig. 6. An exception is the west-side of the $V$-profile. This is the faintest of the profiles and reaches the detection limit already at $\sim 12 \mathrm{kpc}$. Therefore, the outermost data points beyond this distance are not considered further.

\section{Possible systematic errors}

Whilst our detection of the NGC 3957 stellar halo is not limited by photon noise, it may still be affected by a number of systematic errors that we now review and try to quantify. This is of particular importance when trying to interpret the radial changes in the colour profiles.

\subsection{Flat fields}

Errors in flat-fielding may cause artificial gradients in the sky background and, in turn, affect the actual halo colour gradient. Since our skyflats were not taken each night, a possible source of error is a the temporal variability of the flat-fields over a period of several nights. In order to estimate this variability, we compare our normalized $R$-band flat-field obtained at the beginning of the observing run (24 April 2006) with the one corresponding to the end of the observing run (25 May 2006). The changes during this one month period do not exceed $1.5 \%$ across the whole field of view. Since we used the same flat-fields for the sky and the galaxy frames (see Fig. 4), errors in the flat-fields propagate linearly into errors in the final profiles. Consequently, a $1.5 \%$ deviation in the flat-field results in a $1.5 \%$ deviation in the the surface brightness. This translates into a maximum change of shape of $0.01 \mathrm{mag}$, i.e., completely negligible compared with the photon noise.

\subsection{Extended PSF tails}

In a recent work, de Jong (2008) discusses how the extended wings of the point spread function (PSF) can significantly contaminate the measurement of galaxy halo light if not properly accounted for.

By targeting a very low redshift galaxy with a size extending over several arcminutes, we ensured that even the most extended parts of the PSF did not affect the galaxy's surface brightness profiles at all. Indeed, the PSF in our coadded frames has a $F W H M \sim 1$ ", which is more than 100 times smaller than the extent of the measured galaxy halo. For comparison, de Jong (2008) deals with PSFs that are about ten times smaller than the observed galaxy.

In addition, we checked that the wings of the PSF have a limited size. At 1.8 arcsec (corresponding to $0.2 \mathrm{kpc}$ at the distance of NGC 3957) the flux in our PSF drops to $0.72 \%$ of its central intensity in $R$, and to $2.55 \%$ of the central intensity in $V$. The integrated flux of bright standard stars through apertures of growing radius showed that there was no measurable flux in the PSF wings already at $15^{\prime \prime}$ away from the core. Finally, we scaled the PSF to the central intensity of NGC 3957 and measured the flux in its wings. At 50 pixels, i.e., $1.1 \mathrm{kpc}$ from the PSF centre, the level of the flux was below 0.01 ADU, i.e., not measurable. It is clear that the wings of the PSF have no effect on our surface brightness profiles.

\subsection{Difference in the CCD responses: $\alpha$-factors}

The $\alpha$ coefficient described in Sect. 3 (Eq. (2)) accounts for the difference in sensitivity between the two CCDs. The determination of this factor for each single epoch was carried out in two steps.

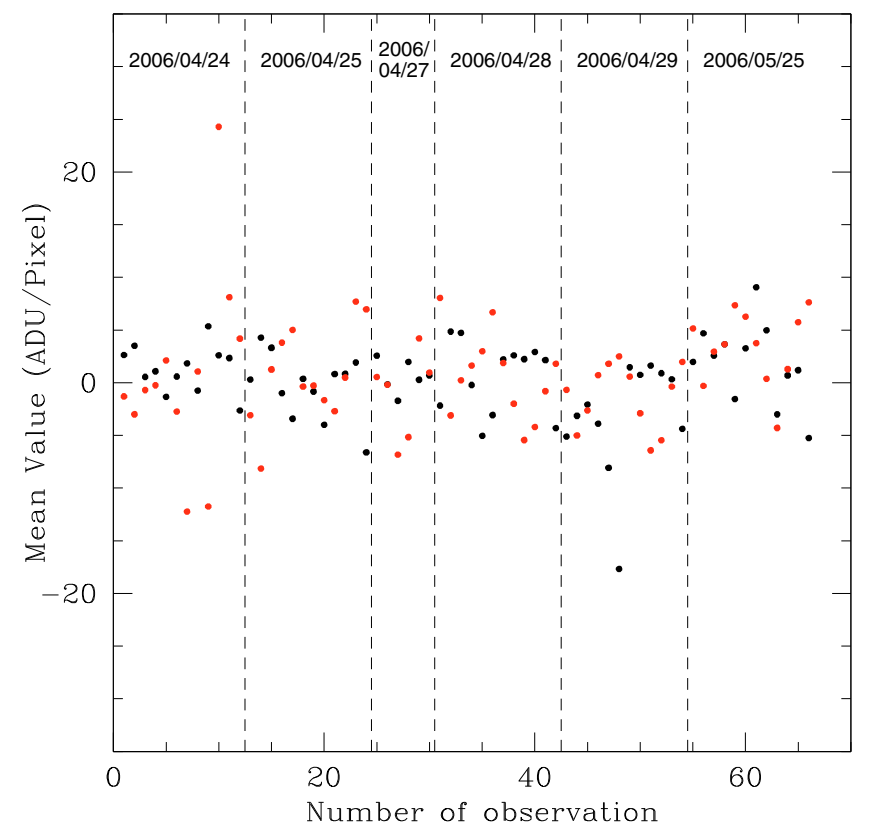

Fig. 7. Mean flux level as measured in a $100 \times 100$ pixel empty box at the boundary of the individual, sky-subtracted frames. The measurements are for the $R$-band. Black circles are for CCD1, while the red circles are for CCD2. There is no systematic difference between CCDs, indicating that $\alpha$ is properly determined.

1- We initialized a first guess value by dividing CCD1 and CCD2 flatfields by pairs.

2- We applied a small correction to this initial value by noticing that the scaling of the sky frames subtracted from CCD1 is proportional to $\alpha$, while the scaling for CCD2 is proportional to $\alpha^{-1}$. As a consequence, a slight error in $\alpha$ is immediately seen as a systematic flux offset between the two CCDs, which is minimal when the value of $\alpha$ is such that the sky subtracts equally well in CCD1 and in CCD2. In order to evaluate this offset and to correct for it, we determined in each individual sky-subtracted frame the mean flux level in a $100 \times 100$ pixels empty region close to the image boundary. Our final measurements are shown in Fig. 7 after correction; there is no residual offset between the mean $\alpha$ factors of CCD1 and CCD2.

The amplitudes of the corrections applied to $\alpha$ were of the order $1 \%$ at most. This translates into changes of about $0.01 \mathrm{mag} / \operatorname{arcsec}^{2}$ in the central regions of the galaxy and to $0.1 \mathrm{mag} / \operatorname{arcsec}^{2}$ beyond $13 \mathrm{kpc}$. The maximum change in slope of the profile, due to an erroneous estimate of $\alpha$, is therefore at most $0.1 \mathrm{mag} / \operatorname{arcsec}^{2}$ in the faintest and most external regions of the galaxy field.

\subsection{Sky-level scaling: $\beta$-factors}

The determination of the mean sky level in each individual image is affected by shot noise, which in turn leads to an over- or under-subtraction through erroneous $\beta$ factors (Eq. (1)). While quantifying this error for each image taken separately is impossible, we used the full data set to estimate the amplitude of the fluctuations with time. This is equivalent to measuring the scatter of the $\beta$ factors for a fixed $\alpha$.

The amplitude of the random fluctuations of the scale factor $\beta$ can be estimated from the scatter of the points in Fig. 7. The standard deviation of the $132 R$-band images is $4.66 \mathrm{ADU}$, 
P. Jablonka et al.: Direct detection of galaxy stellar halos
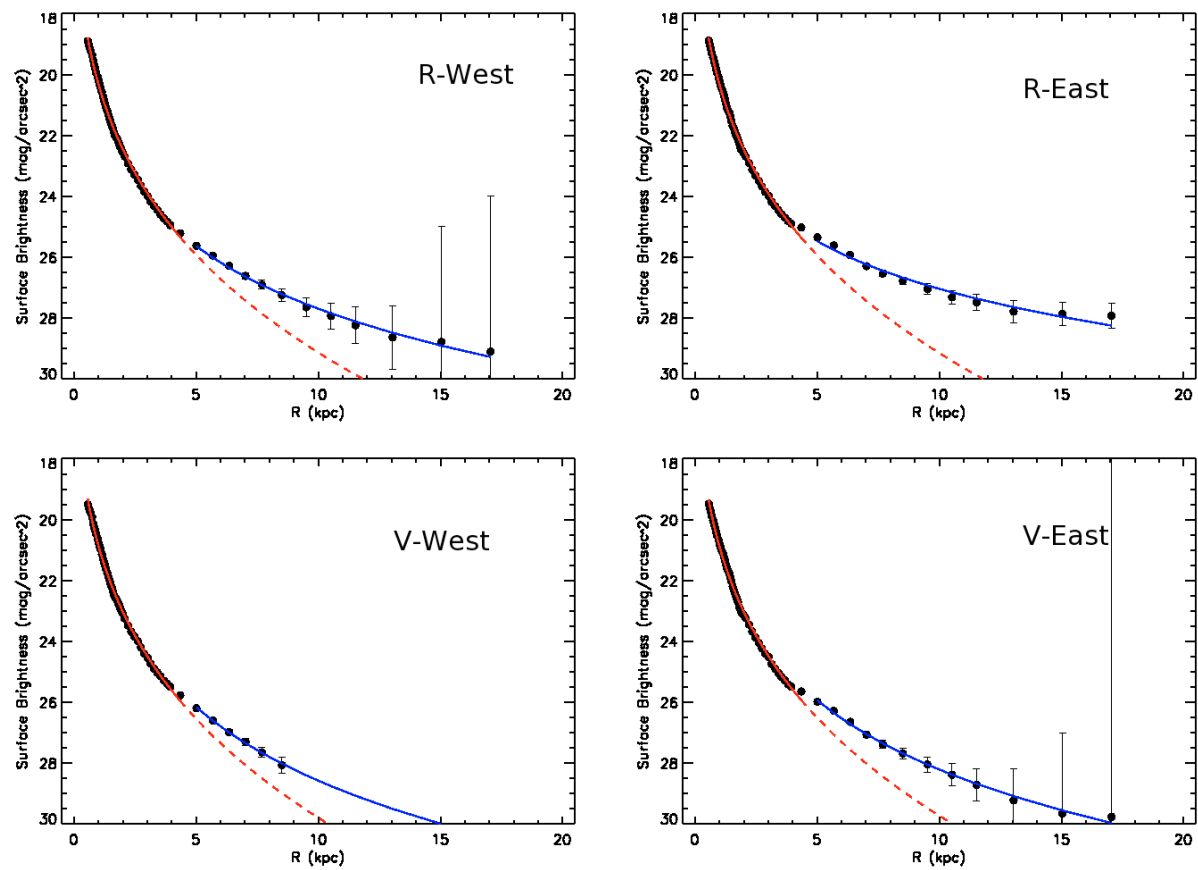

Fig. 8. The two-component fits to the minor axis profiles in the $V$ and $R$-bands. The observed profiles are shown with solid dots with attached error bars (see Sect. 5). The last point of the $\mathrm{V}$-West profile is not shown, neither is it used in the fits, because it is obviously dominated by errors (see Sect. 4). The red lines indicate the de Vaucouleurs law, which best fits the bulge of NGC 3957. The red solid line indicates the region of the fits within $5 \mathrm{kpc}$, while the dashed line shows its extrapolation to larger radii. The blue lines show the power-law component of the fit at radii larger than $5 \mathrm{kpc}$. while the uncertainty on the mean $\beta$ is 0.41 ADU. Similarly, the standard deviation of the $50 \mathrm{~V}$-band frames is $3.75 \mathrm{ADU}$, while the uncertainty of the mean value of $\beta$ is $0.53 \mathrm{ADU}$. These values provide good estimates of an upper limit on the errors on $\beta$ in $V$ and $R$.

A systematic error on $\beta$ may have important consequences on the colour profile of the galaxy, in particular if the amplitude of the error is different in $V$ and in $R$. In order to estimate this effect, we artificially introduced an over- or under-subtraction of the sky by $2 \sigma$ in both bands. This corresponds to a shift of $2 \times 0.41 \approx 0.8$ ADU in $R$ and $2 \times 0.53 \approx 1.1 \mathrm{ADU}$ in $V$.

It translates into a variation of $\sim 0.05 \mathrm{mag}$ at $25.5 \mathrm{mag} \operatorname{arcsec}^{2}, \sim 0.2 \mathrm{mag}$ at $27 \mathrm{mag} \operatorname{arcsec}^{2}$ and $\sim 0.5 \mathrm{mag}$ at $28 \mathrm{mag} \operatorname{arcsec}^{2}$ in the $R$-band. Similarly, the shifts in the $V$-band are $\sim 0.05 \mathrm{mag}, \sim 0.4 \mathrm{mag}$, and more than $\sim 1.0 \mathrm{mag}$ at 26,28 , and 30 mag $\operatorname{arcsec}^{2}$, respectively.

\subsection{Extended halo}

The last possible source of error is the presence of a very extended halo signal in the $\mathrm{SKY} 1_{\mathrm{i}}$ and $\mathrm{SKY} 2_{\mathrm{i}}$ frames used to scale MS2 and MS1 before subtraction (see Fig. 4). This rescaling indeed assumes that the CCD frames, which do not contain NGC 3957, are far enough away from the galaxy to be free from any residual halo light.

Since the reduction and scaling procedures are the same in the $V$ and $R$ filters, the systematics causing a positive (negative) shift in $V$ would also cause a positive (negative) shift in $R$. From their radial minor axis profiles of M 31, Irwin et al. (2005) derive a power law surface brightness profile following $I(r) \propto r^{-2.3}$, beyond $20 \mathrm{kpc}$. We used this relation to evaluate to what extent $S K Y 1_{i}$ and $S K Y 2_{i}$ might be contaminated by extended halo light from one CCD on the other. Our sky frames are located at a minimum of twice the distance from the galaxy centre to the edge of our galaxy profile. Assuming the Irwin et al. (2005) M 31 halo surface brightness can be considered representative, the halo light in the CCDs we used to measure the sky should be at least five times fainter than at the edge of the CCD that contains the galaxy.

This translates into an error of $1 \mathrm{ADU} /$ pixel, which is a very conservative value, corresponding to about $40 \%$ of the measured $R$-band flux and $60 \%$ of the flux in the $V$-band at $13 \mathrm{kpc}$ from the galaxy centre. This modifies the $V$ and $R$ East-side magnitudes by $\sim 0.03 \mathrm{mag}$ at $5 \mathrm{kpc}, \sim 0.2 \mathrm{mag}$ at $10 \mathrm{kpc}$, and $\sim 0.3-0.4$ mag $(R)$ respectively $\sim 0.5 \mathrm{mag}(V)$ at $15 \mathrm{kpc}$.

\subsection{Total error budget}

To summarize, the main possible sources of errors that can affect the $V$ and $R$ surface brightness profiles of NGC 3957 presented here are i) the uncertainties related to the scaling of the sky-level through the $\beta$-factors and ii) the possible overestimation of the actual sky background level, caused by contamination by a genuine extended stellar halo signal in the regions used to build the sky frames. This latter factor can only act as sky over-subtraction and therefore will contribute only to the upper limit of the error bars. Our surface brightness profiles are presented in Fig. 8, along with their total error bars.

\section{Properties of the stellar halo}

\subsection{Detection in $V$ and $R$}

As shown in Fig. 6, we clearly detect light up to about $15 \mathrm{kpc}$ away from the centre of NGC 3957. In order to evaluate the structure of this luminous component and, more importantly, to see whether one can resolve it into more than a single structure, we performed $1 \mathrm{D}$-fits to the $V$ and $R$ profiles. We considered only the photon noise error bars, since they are the only statistically meaningful quantities to be taken into account in $\chi^{2}$ procedures. We used the IDL routine mpfit, which solves the least-squares problem with the Levenberg-Marquardt technique, to conduct the fits. The inner $0.5 \mathrm{kpc}$ region was not considered due to the presence of the dust lane. We first started with a de Vaucouleur law form, chosen to best represent the bulge of 


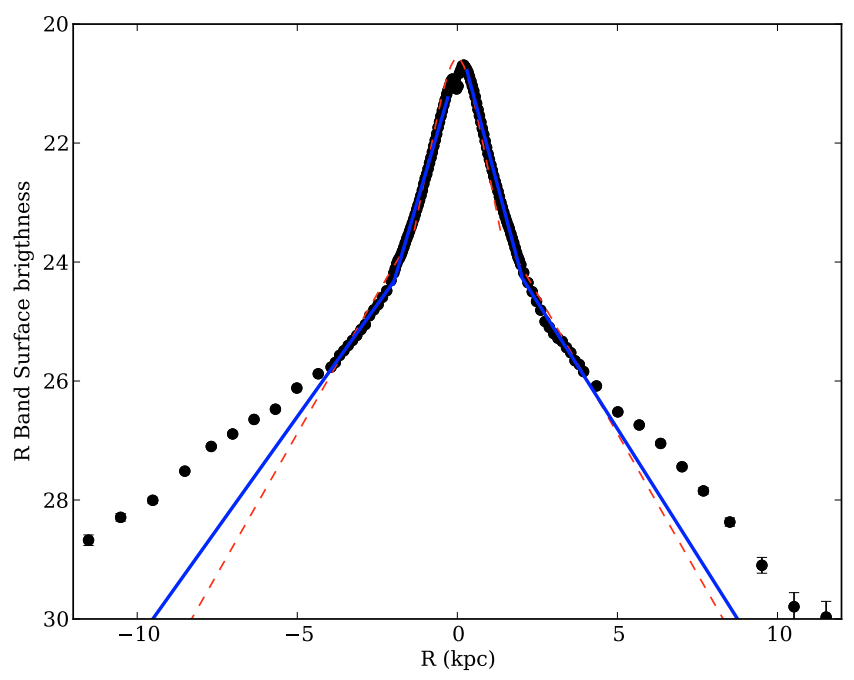

Fig. 9. The $R$-band vertical profile of NGC 3957 extracted at a position $5 \mathrm{kpc}$ along the major axis. The observations are displayed with open circles. The Pohlen et al. (2004) fits of the thin and thick discs are indicated with a dashed red line, while our pure exponential fits are shown with a plain blue line.

this S0 galaxy. Figure 8 shows that while a $r^{1 / 4}$ function with an effective radius of $0.29 \mathrm{kpc}$ provides an excellent description of the inner profile in both in $V$ and $R$ of NGC 3957, it considerably under-predicts the light beyond $4-5 \mathrm{kpc}$. Beyond $5 \mathrm{kpc}$, the excess light is well-represented by a power-law with index $-2.76 \pm 0.43$. A pure exponential fit with a scale length of a few kpc always provides a worse fits, particularly in the $R$ band, for which it was essentially discarded. Ibata et al. (2009) applied a de Vaucouleur law with an effective radius of $1.55 \mathrm{kpc}$ to NGC 891's extra-planar light, but this did not provide a good description of the light detected here.

Pohlen et al. (2004) present 3D thick/thin disc decompositions for a sample of eight S0 galaxies, including NGC 3957. They derive an $R$-band vertical scale height for the thick disc component of $2.3 \mathrm{kpc}$, which, by their definition, corresponds to a normal exponential vertical scale height of $1.65 \mathrm{kpc}$. In order to compare this value with our own, we needed to extract a vertical profile some distance from the minor axis in order to eliminate the influence of the bulge. Figure 9 shows the $V$ and $R$ West and East profiles of NGC 3957 extracted at a position $5 \mathrm{kpc}$ along the major axis. Fitting the thick disc between 2 and $4 \mathrm{kpc}$, we found an exponential scale height of $1.27 \mathrm{kpc}$, which agrees well with that found by Pohlen et al. (2004), considering the different ways in which the two studies have extracted profiles and their different photometric depths and spatial resolutions. This fit and also that from Pohlen et al. (2004) are overlaid in the figure. Figure 9 also underscores the need for an additional component beyond a thick disc at vertical distances greater than $\sim 4 \mathrm{kpc}$.

\subsection{Colour profile}

Figure 10 displays the minor-axis colour profile of NGC 3957. The apparent strong reddening at large galactocentric distances must be analysed in light of the possible systematic errors presented in Sect. 5. As seen in Fig. 6, the NGC 3957 surface brightness profile is traced down to about 1.2 mag above the detection limit both in $V$ and $R$ bands.

We now analyse the west side of the galaxy (negative values of the radii) as an illustration of the different elements at play.

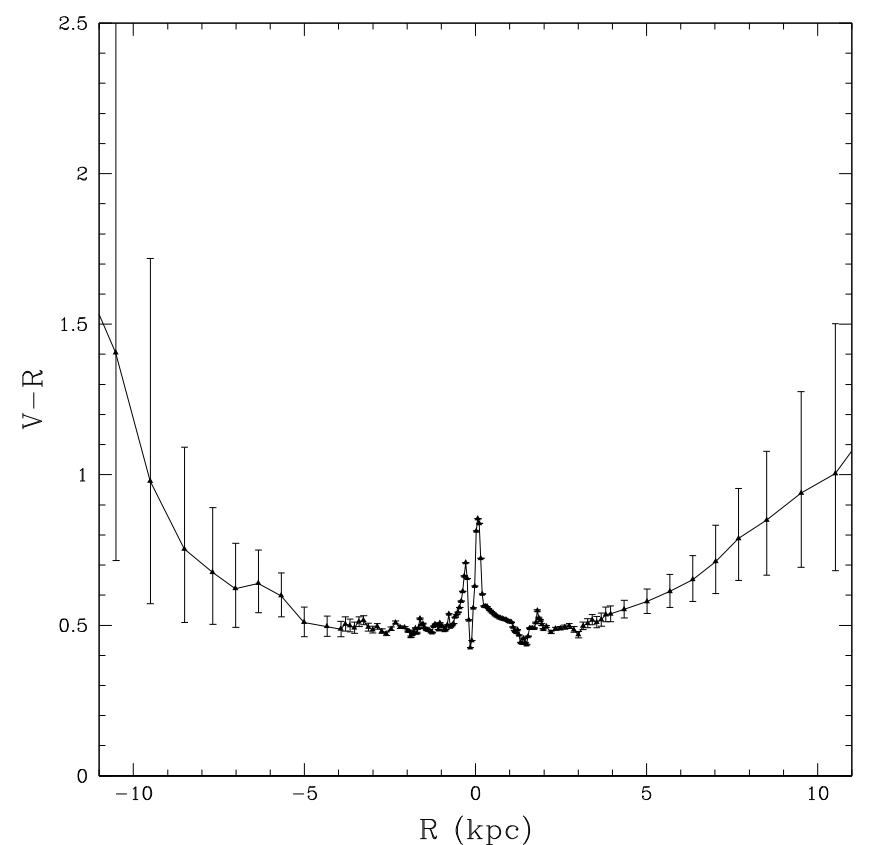

Fig. 10. The $V-R$ colour profile of NGC 3957. The edges of the vertical bars show the maximum possible systematic errors, which dominate over the photon noise, as estimated from the uncertainties in the skylevel scaling $\beta$ factors (Sect. 5.4) and considering possible extended halo (Sect. 5.5).

The total error bars in $V$ and $R$ are small and similar from the galaxy centre, up to about $8.5 \mathrm{kpc}(\Delta \leq 0.05 \mathrm{mag})$. At $9.5 \mathrm{kpc}$, the error in $V$ is 0.2 mag larger than the one in $R$. This difference keeps increasing with the radius, reaching 0.95 mag at $10.5 \mathrm{kpc}$. This effect is due to the strong decrease in $V$-flux rendering the sky subtraction very critical, i.e., a given error on the sky subtraction has much larger impact on the $V$-band fainter profile than on the $R$-band one. To be quantitative, at $8.5 \mathrm{kpc}$, the $V$ and $R$ fluxes are at the level of $\sim 4$ ADU/pixel and $~ 4.5$ ADU/pixel, respectively. At $9.5 \mathrm{kpc}$, we measure only $\sim 2 \mathrm{ADU} /$ pixel and $\sim 3.5 \mathrm{ADU} /$ pixel in $V$ and $R$, respectively. Figure 10 clearly shows that the larger the error bars due to systematics, the steeper the rise in $V-R$. This suggests that this reddening may not be a genuine property of the stellar/dust properties of NGC 3957 halo, but rather an artifact due to the sharp drop in the $V$ flux. The sky subtraction becomes very uncertain and leads to biased results when the halo surface brightness approaches the detection limit by less than two magnitudes, as indicated in Fig. 6. This is the case at a distance of $10 \mathrm{kpc}$ from the galaxy centre in $V$, and at $15 \mathrm{kpc}$ in the $R$-band.

The west-side mean colour profile has $V-R \sim 0.50 \pm 0.02$ from $2 \mathrm{kpc}$ from the centre of the galaxy, up to $5 \mathrm{kpc}$ and then marginally rises to $0.70 \pm 0.2$, at $8 \mathrm{kpc}$. As discussed earlier, the error bars are not independent from each other and rather reflect systematic errors. They all act in the same direction, either increasing or decreasing the overall $V-R$ profile. The upper boundary (i.e., connecting the upper limits of the error bars) corresponds to an error of $1 \mathrm{ADU} / \mathrm{pix}$ on the true sky level, or equivalently to a $3 \sigma$ error (1.2 and 1.6 ADU in $R$ and $V$, respectively) on the scaling of the sky. Although very modest, these values have noticeable consequences on the shape of the profiles, due to the faint luminosity levels considered here. In regions where the $V-R$ gradient is steeper than $0.1-0.2$ mag per kpc, the systematic errors dominate. The analysis of the east side 
of NGC 3957 follows the same line, albeit the mean colour is redder by $\sim 0.05 \mathrm{mag}$ with respect to the west-side, even in the regions where the systematic errors are negligible. The small colour asymmetry between the east and west sides of NGC 3957 is intriguing and could possibly reflect fluctuations due to substructures within the galaxy's halo.

We now investigate whether the inner halo colour of NGC 3957 is indeed compatible with those of normal stellar populations, and conduct comparisons with a few galaxies for which the halo stellar population could be resolved and analysed. We decomposed the halo population into the same number of single stellar populations (SSPs) as there are bins in the published metallicity distribution function (MDF). These SSPs were generated with metallicities equal to that of the bin mean using the $\mathrm{Y}^{2}$ isochrones of Kim et al. (2002), that extend down to $[\mathrm{Fe} / \mathrm{H}]=-3.8$, with $[\alpha / \mathrm{Fe}]=0.3$, a Salpeter IMF and a $13 \mathrm{Gyr}$ age. When necessary the isochrones were interpolated to represent the metallicities of the MDFs. The resultant SSPs were then summed in luminosity, weighted by the fraction of light contributed by each MDF bin.

We first derived the expected integrated $V-R$ colour of the Milky Way halo from the metallicity distribution of Carollo et al. (2010). They find that from a vertical distance of $5 \mathrm{kpc}$ up to $9 \mathrm{kpc}$ above the Galactic plane, which corresponds to the regions we sampled in the present study, the halo stellar metallicities span $[\mathrm{Fe} / \mathrm{H}]=-3.0$ to -1 with a small fraction of stars reaching $[\mathrm{Fe} / \mathrm{H}]=-0.5$. The distributions at $5.5 \mathrm{kpc}$ and $8.5 \mathrm{kpc}$, peaking at $[\mathrm{Fe} / \mathrm{H}]=-1.5$ at and $[\mathrm{Fe} / \mathrm{H}]=-2$, respectively, lead to $V-R=0.49$ and 0.47 . This narrow range of colours is due to the progressive insensitivity of the isochrones to changes in metallicity at low $[\mathrm{Fe} / \mathrm{H}]$.

As to M 31, the complex web of stellar streams prevented us from deriving a priori a unique colour for its halo. We instead examined the possible range of values based on earlier works providing complete metallicity distributions. The metallicity distribution derived by Ibata et al. (2007) from the diffuse light of M 31 halo translates into $V-R \sim 0.51$. The M2 field of Durrell et al. (2001), located at $20 \mathrm{kpc}$ along the M 31 minor axis, peaks at $[\mathrm{M} / \mathrm{H}] \sim-0.5$ with a long tail of more metal-poor population down to -2.5 , leading to an integrated $V-R \sim 0.54$. In contrast, the spectroscopic study of Koch et al. (2008) reveals a strong metallicity gradient with a peak at $[\mathrm{Fe} / \mathrm{H}] \sim-1$. for galactocentric distances between $20 \mathrm{kpc}$ and $40 \mathrm{kpc}$ and at $[\mathrm{Fe} / \mathrm{H}] \sim-2$ beyond $40 \mathrm{kpc}$, with $V-R \sim 0.47$.

Now turning to slightly more distant galaxies Mouhcine et al. (2007) analyse the 1.5 first magnitude of the RGB stars in the halo NGC 891, at $\sim 9.5 \mathrm{kpc}$ from the galactic plane, centred on $V-I \sim 1.5$ and derive a peak metallicity of $[\mathrm{Fe} / \mathrm{H}] \sim-1$, i.e., close to an integrated colour of $V-R \sim 0.5$. Some of the galaxies in the sample of Mouhcine et al. (2005a) have metallicity distribution peaks at $[\mathrm{M} / \mathrm{H}] \sim-0.6$. Their full metallicity distributions results in an integrated $V-R \sim 0.54$.

In summary, $V-R$ from $\sim 0.45$ to $\sim 0.6$ constitutes the range of expected integrated colours of old and preferentially metalpoor galactic halos. The halo of NGC 3957, with $V-R$ from 0.5 to $0.7 \mathrm{mag}$, between 5 and $8 \mathrm{kpc}$ from the galaxy centre, where the stellar halo component is clearly dominating and the systematic errors are still modest, agrees fairly well with these numbers. Beyond these vertical distances, the colour reddening correlates with increasing errors. The order magnitude of the former being similar or smaller than that of the latter suggests that the colour gradient is due to the uncertainties in sky subtraction at very faint flux levels.

\section{Conclusions}

We obtained ultra-deep optical VLT/VIMOS images of NGC 3957, a nearby edge-on S0 galaxy. The total exposure time of six hours in $R$ and $7 \mathrm{~h}$ in $V$ allowed us to reach the limiting magnitudes of $R=30.6 \mathrm{mag} / \operatorname{arcsec}^{2}$ and $V=31.4 \mathrm{mag} / \operatorname{arcsec}^{2}$ in the Vega system.

We devised a new observational strategy based on infrared techniques, which takes advantage of the large field of view of VIMOS. By observing NGC 3957 alternatively in two different CCDs, we were able to create high signal-to-noise and stable sky frames, which were used to carry out accurate sky subtraction, without any assumption on the spatial shape and flux level of the sky background. The observations allowed us to reach unprecedented high signal-to-noise ratios up $15 \mathrm{kpc}$ away from the galaxy centre, rendering photon-noise negligible. They enabled the clear detection of the stellar halo of NGC 3957 at distances above $5 \mathrm{kpc}$.

We conducted a thorough analysis of the possible sources of systematic error that could affect the vertical surface brightness and colour profiles: flat-fielding, differences in CCD responses, scaling of the sky background, extended halo, PSF wings. While a $r^{1 / 4}$ function with an effective radius of $0.29 \mathrm{kpc}$ provides an excellent description of the inner $V$ and $R$-band minor axis profiles of NGC 3957, it under-predicts the light beyond 4-5 kpc. Above $5 \mathrm{kpc}$, the galaxy light profile requires an additional power-law component with index $-2.76 \pm 0.43$.

The most secure part of the NGC 3957 halo colour profile falls in the range $V-R \sim 0.5-0.7 \mathrm{mag}$. This colour is compatible with the properties of nearby galaxy halos as revealed by the investigation of their resolved stellar populations and with that of the Milky Way. An apparent strong reddening is seen in the outer parts ( $r \gtrsim 8 \mathrm{kpc}$ ) of the $V-R$ profile, similarly to earlier works on galaxy halo surface brightness profiles. However, our analysis indicates that this reddening may not be a genuine property of the stellar/dust properties of NGC 3957 halo, and simply reflects the impact of systematic errors in sky subtraction at extremely faint flux levels. It is possible that previously published reports of extremely red colours in external galaxy halos could have resulted from similar effects.

Future imaging programmes benefiting from larger fields of view $(\sim 0.5-1.0$ degree on one side) will allow the systematics in halo emission searches to be controlled even further. Existing facilities to carry out the experiment include the SuprimeCam on the Subaru telescope and the VST (VLT survey telescope).

Acknowledgements. This research used the facilities of the Canadian Astronomy Data Centre operated by the National Research Council of Canada with the support of the Canadian Space Agency. This research is partially supported by the Swiss National Science Foundation (SNSF). AMNF is supported by a Marie Curie Excellence Grant from the European Commission under contract MCEXT-CT-2005-025869.

\section{References}

Abadi, M. G., Navarro, J. F., \& Steinmetz, M. 2006, MNRAS, 365, 747 Barker, M. K., Ferguson, A. M. N., Irwin, M., Arimoto, N., \& Jablonka, P. 2009, AJ, 138, 1469

Bell, E. F., Zucker, D. B., Belokurov, V., et al. 2008, ApJ, 680, 295 Belokurov, V., Evans, N. W., Irwin, M. J., et al. 2007, ApJ, 658, 337 Bullock, J. S., \& Johnston, K. V. 2005, ApJ, 635, 931

Carollo, D., Beers, T. C., Chiba, M., et al. 2010, ApJ, 712, 692 de Jong, R. S. 2008, MNRAS, 388, 1521

Durrell, P. R., Harris, W. E., \& Pritchet, C. J. 2001, AJ, 121, 2557

Eggen, O. J., Lynden-Bell, D., \& Sandage, A. R. 1962, ApJ, 136, 748 Ferguson, A. M. N., Irwin, M. J., Ibata, R. A., Lewis, G. F., \& Tanvir, N. R. 2002, AJ, 124, 1452 
Font, A. S., Johnston, K. V., Bullock, J. S., \& Robertson, B. E. 2006, ApJ, 638, 585

Gilmore, G., Wyse, R. F. G., \& Norris, J. E. 2002, ApJ, 574, L39

Grillmair, C. J. 2006, ApJ, 645, L37

Ibata, R. A., Gilmore, G., \& Irwin, M. J. 1994, Nature, 370, 194

Ibata, R., Irwin, M., Lewis, G., Ferguson, A. M. N., \& Tanvir, N. 2001, Nature, 412,49

Ibata, R., Martin, N. F., Irwin, M., et al. 2007, ApJ, 671, 1591

Ibata, R., Mouhcine, M., \& Rejkuba, M. 2009, MNRAS, 395, 126

Irwin, M. J., Ferguson, A. M. N., Ibata, R. A., Lewis, G. F., \& Tanvir, N. R. 2005, ApJ, 628, L105

Jurić, M., Ivezić, Ž., Brooks, A., et al. 2008, ApJ, 673, 864

Kim, Y., Demarque, P., Yi, S. K., \& Alexander, D. R. 2002, ApJS, 143, 499

Koch, A., Rich, R. M., Reitzel, D. B., et al. 2008, ApJ, 689, 958

Lequeux, J., Fort, B., Dantel-Fort, M., Cuillandre, J.-C., \& Mellier, Y. 1996, A\&A, 312, L1

Lequeux, J., Combes, F., Dantel-Fort, M., et al. 1998, A\&A, 334, L9

Majewski, S. R., Siegel, M. H., Kunkel, W. E., et al. 1999, AJ, 118, 1709

Martínez-Delgado, D., Peñarrubia, J., Gabany, R. J., et al. 2008, ApJ, 689, 184

Martínez-Delgado, D., Pohlen, M., Gabany, R. J., et al. 2009, ApJ, 692, 955
McConnachie, A. W., Irwin, M. J., Ibata, R. A., et al. 2009, Nature, 461, 66 Mouhcine, M., Ferguson, H. C., Rich, R. M., Brown, T. M., \& Smith, T. E. 2005a, ApJ, 633, 821

Mouhcine, M., Rich, R. M., Ferguson, H. C., Brown, T. M., \& Smith, T. E. 2005b, ApJ, 633, 828

Mouhcine, M., Rejkuba, M., \& Ibata, R. 2007, MNRAS, 381, 873

Newberg, H. J., Yanny, B., Rockosi, C., et al. 2002, ApJ, 569, 245

Pohlen, M., Balcells, M., Lütticke, R., \& Dettmar, R.-J. 2004, A\&A, 422, 465

Rejkuba, M., Mouhcine, M., \& Ibata, R. 2009, MNRAS, 396, 1231

Richardson, J. C., Ferguson, A. M. N., Johnson, R. A., et al. 2008, AJ, 135, 1998 Rocha-Pinto, H. J., Majewski, S. R., Skrutskie, M. F., \& Crane, J. D. 2003, ApJ, 594, L115

Sackett, P. D., Morrison, H. L., Harding, P., \& Boroson, T. A. 1994, Nature, 370, 441

Schlegel, D. J., Finkbeiner, D. P., \& Davis, M. 1998, ApJ, 500, 525

Tanaka, M., Chiba, M., Komiyama, Y., et al. 2010, ApJ, 708, 1168

Vivas, A. K., \& Zinn, R. 2006, AJ, 132, 714

Zheng, Z., Shang, Z., Su, H., et al. 1999, AJ, 117, 2757

Zibetti, S., \& Ferguson, A. M. N. 2004, MNRAS, 352, L6

Zibetti, S., White, S. D. M., \& Brinkmann, J. 2004, MNRAS, 347, 556 